\title{
ARQUITETURA PARA INTEGRAÇÃO DE SERVIÇOS VIA API REST PARA PLATAFORMA DE ACERVOS DIGITAIS
}

\author{
Larissa Herculano, Matheus de Carvalho Sobrinho, Dário Pereira dos Santos, \\ Ariovaldo Dias Furtado, Lucimar Rizzo Lopes dos Santos e Daniel Alves da Silva \\ Universidade de Brasília - Departamento de Engenharia Elétrica, Brasília, DF, Brasil - Zipcode 70910-900
}

\begin{abstract}
RESUMO
Arquiteturas descentralizadas vêm crescendo bastante nos últimos anos com a ideia de desacoplar sistemas monolíticos para facilitar a comunicação entre os clientes e os serviços presentes neste tipo de ambiente. Contudo, os padrões da arquitetura de microsserviços ainda necessitam de um modelo mais completo e que defina todas as etapas da sua implantação. Este trabalho propõe um modelo de arquitetura para implementação de módulos que, acoplados ao DSpace versão 6, possibilitem a manipulação de metadados de itens digitais de forma a minimizar o tempo de inserção na ferramenta, isso é feito a partir de uma otimização no processo de inserção atual do DSpace. Os resultados obtidos com a integração do módulo de submissão, proporcionou um ganho na produtividade dos usuários submissores do sistema.
\end{abstract}

\section{PALAVRAS-CHAVE}

Repositório Digital, Arquitetura de Serviços, API Rest

\section{INTRODUÇÃO}

Diante da iniciativa de acesso aberto e disponibilização de acervos em rede, a implantação de repositórios digitais tem sido amplamente empregada por diversas instituições. Entre os sistemas de gerenciamento de acervos digitais mais utilizados estão as plataformas E-Prints e DSpace. A plataforma E-Prints foi designada inicialmente para a criação de repositórios institucionais de pré-prints para pesquisa acadêmica, porém foi expandida para uso de diversos materiais, inclusive relatórios técnicos, publicações em conferências e outros. A plataforma DSpace consiste na aplicação mais popular para gerenciamento de bibliotecas digitais, projetada para capturar, armazenar, indexar e disponibilizar recursos informacionais em diversos formatos (Wei, 2011).

Em projeto de pesquisa aplicada em cooperação com o Ministério da Economia (ME) planejada a construção do repositório, intitulado Biblioteca Digital do ME, optando pela ferramenta DSpace, por ser mais comumente utilizada no Brasil e pela comunidade internacional. O acervo contemplado no repositório engloba documentos com características distintas, dividindo-se em dois segmentos, a saber: publicação e legislação. De acordo com as características do acervo, foram elencados metadados específicos para cada um destes segmentos. Os metadados são registros de dados para identificação do recurso informacional disponibilizado. Segundo Viana, Márdero Arellano e Shintaku (2005), os metadados fornecem significado semântico a dados supostamente isolados, proporcionando contexto para o processo de busca, o que expande a relevância dos resultados por meio da padronização na descrição dos recursos. O repositório demandou adaptações para atender com maior eficiência às necessidades do acervo. Visando benefícios nos processos de trabalho com a ferramenta, foi desenvolvido um formulário de inserção para cada segmento.

O segmento do acervo intitulado publicação, abrange documentos como relatórios, planos, periódicos, apresentações, cartilhas, trabalhos acadêmicos, entre outros. Para esse segmento foi criado o formulário "A", com os metadados elencados para atender às suas características específicas. O segmento do acervo denominado legislação contempla leis, decretos, portarias, despachos, entre outros. Para esse segmento, o formulário "B" foi elaborado, com metadados diferenciados considerando suas particularidades.

O trabalho desenvolvido com a ferramenta modelada permitiu que as etapas de submissão padrão do software DSpace fossem reduzidas, a fim de otimizar a atividade de inserção. Permitiu ainda, que itens de uma mesma coleção compartilhassem metadados, de acordo com o formulário selecionado, atendendo aos requisitos indicados pelos profissionais da informação, bibliotecários da instituição. 


\section{TRABALHOS RELACIONADOS}

O uso da ferramenta DSpace tem-se tornado constante entre várias instituições públicas, privadas e de terceiro setor para a estruturação de repositórios digitais. Suas características favorecem a escolha, pois trata-se de um software livre, amplamente customizável e com código aberto. Apesar de ser uma das ferramentas mais utilizadas, nem sempre corresponde com exatidão às necessidades das instituições. Shintaku e Vechiato (2018) demonstram que instituições no Brasil apresentam necessidades além das atendidas pela instalação padrão do DSpace, o que demanda extensões das funcionalidades originalmente disponibilizadas pela ferramenta. Os autores ressaltam ainda que é escasso o compartilhamento das funcionalidades desenvolvidas, resultando na necessidade de maior disseminação e interação entre as equipes de tecnologia da informação que atuam com DSpace no Brasil. Afirmam que o Brasil é ativo na evolução da ferramenta, pois dispõe de numerosas instalações e iniciativas de extensão das funcionalidades padrão.

Behr e Ferreira (2016) relatam a experiência de customização da ferramenta DSpace, no repositório digital da Universidade Federal do Rio Grande do Sul (UFRGS), denominado Lume, na qual foram implantadas melhorias em relação ao armazenamento de registros de acessos e downloads visando a correta geração de relatórios estatísticos, e ainda em relação à submissão de arquivos e notificação por e-mail de entradas recentes, que por sua vez, objetivam aperfeiçoar a interação do usuário com o sistema.

Outra implementação no DSpace, ainda no repositório Lume, foi realizada por Costa et al. (2015) com o intuito de criação da etapa de revisão técnica no processo de submissão de itens na comunidade de recursos educacionais (RE). Com a modificação proposta, os autores buscaram garantir o acesso propriamente pelo navegador, dispensando instalação prévia, para maior agilidade no processo de revisão, disponibilização e acesso pelos usuários do repositório.

\section{DESCRIÇÃO DO PROBLEMA E MOTIVAÇÃO}

Diante do excessivo número de documentos que compõem o acervo da biblioteca do ME, observou-se que, para a disponibilização, seria indispensável copioso esforço na plataforma pois a inserção deve ser realizada item a item. Assim sendo, o formulário padrão contido no sistema DSpace não proporciona eficiência, tendo em vista a necessidade de cinco tarefas para a efetivação do processo de submissão, entre as quais duas referem-se à descrição do item, uma refere-se ao arquivo a ser anexado, uma outra refere-se à verificação dos dados inseridos e a última refere-se à licença atribuída ao item. Verifica-se as tarefas na Figura 1.

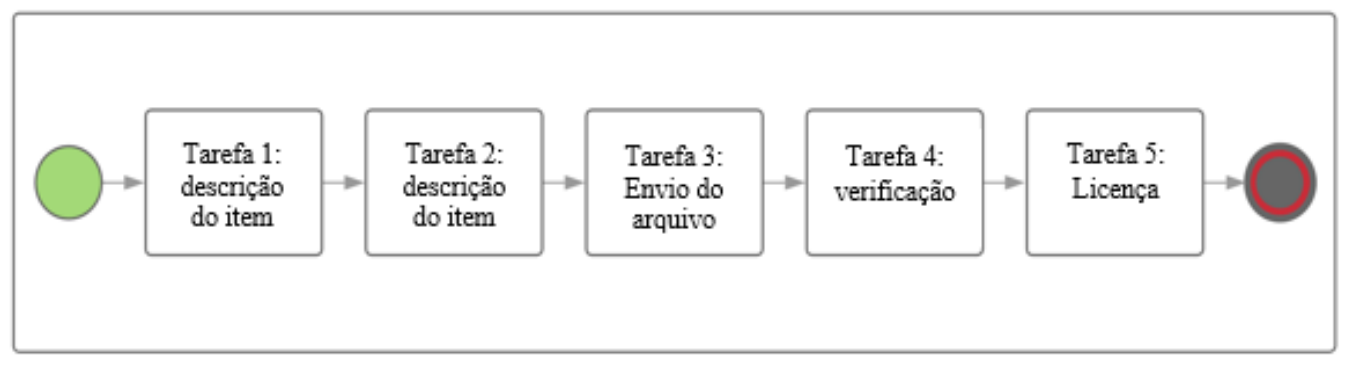

Figura 1. Modelo de processo de inserção padrão (fonte: elaboração do autor)

Ademais, para atender adequadamente ao acervo seriam necessários dois tipos de formulário, com os metadados distintos determinados respectivamente no formulário " $A$ " para o segmento publicação e no formulário "B" para o segmento legislação. Nesse contexto, outro problema identificado foi a integração entre os usuários e os administradores de sistema. No caso de mais de um tipo de formulário disponível, a cada nova coleção criada, é preciso acionar o administrador para que ele faça manualmente o mapeamento da nova coleção para o formulário que deve ser apresentado no momento da inserção de um novo item digital.

A equipe de pesquisadores de ciências e tecnologia da informação, foram motivadas a desenvolver uma solução que pudesse minimizar os problemas identificados, no que tange a este contexto específico, possibilitando uma melhoria tanto para o sistema, quanto para os usuários, facilitando o trabalho diário dos bibliotecários e agilizando o processo de inserção na ferramenta. 


\section{BIBLIOTECA DIGITAL DO MINISTÉRIO DA ECONOMIA}

A Biblioteca Digital é o testemunho da memória institucional, criada com o intuito de reunir, armazenar, organizar, preservar, recuperar e disseminar a informação gerada na produção do conhecimento pelas unidades integrantes do ME. Destacam-se os objetivos principais: preservar a informação governamental, ampliar a visibilidade de forma a alcançar maior número de usuários; promover maior rapidez na recuperação de informações do Governo Federal; promover a interoperabilidade de conteúdo digital com outras redes de informação; e incentivar a produção de publicações em diversos formatos digitais.

A implantação da Biblioteca Digital do ME tem gerado vantagem para a realidade da instituição, bem como a customização e implementação da ferramenta DSpace tem apontado caminhos para a gestão da informação e acesso ao conteúdo digital institucional. Inicialmente customizada na linguagem XMLUI ${ }^{1}$ e após estudos, atualizada para JSPUI $^{2}$, tendo em vista as melhorias e interface de usuário mais moderna e dinâmica apresentada pela linguagem.

A criação do projeto, customização e implantação da Biblioteca Digital do ME foi um trabalho realizado pela equipe do Laboratório de Tecnologias da Tomada de Decisão (Latitude), da Faculdade de Tecnologia (FT), da Universidade de Brasília (UnB) em virtude do Termo de Cooperação Técnica, firmado entre o ME e a UnB.

\subsection{Organização da Informação no Framework DSpace}

A organização da informação na ferramenta DSpace é feita com a construção de uma estrutura informacional hierárquica de navegação, constituída por comunidades, subcomunidades e coleções. Na Biblioteca Digital do ME a estrutura foi fundamentada nas unidades integrantes do órgão e a inserção dos itens digitais ocorreu de acordo com os assuntos relacionados às atividades de cada unidade, conforme desenho elaborado e validado pelos profissionais da biblioteca da instituição em conjunto com os pesquisadores da UnB.

O número crescente de itens digitais contempla documentos de forma a refletir a história da produção intelectual do órgão, com informações relevantes para a gestão do conhecimento e publicidade de informações relevantes à sociedade. Contudo, para que seja atingida a eficácia na gestão do conhecimento e na publicidade de informações, é necessário que haja uma boa indexação dos itens digitais, com a escolha correta dos metadados a serem utilizados.

O padrão de metadados utilizado pelo DSpace é o Dublin Core (DC), da Dublin Core Metadata Initiative DCMI (Weibel e Koch, 2000), que permite escolha de cada elemento e qualificador, opcionalmente, para que a descrição seja adaptada às necessidades do tipo de recurso informacional em questão. O padrão DC é descrito na norma ISO 15836-1:2017, "que estabelece 15 elementos principais de metadados para a descrição de recursos entre domínios. Esses termos fazem parte de um conjunto maior de vocabulários de metadados mantidos pela Dublin Core Metadata Initiative" (ISO, 2017, tradução nossa). Utiliza para definição do padrão do campo data, a norma ISO 8601-1:2019, que "especifica representações adicionais de datas do calendário gregoriano e horários com base no relógio de 24 horas bem como elementos compostos deles, como cadeias de caracteres para uso no intercâmbio de informações" (ISO, 2019, tradução nossa), com o formato AAAA-MM-DD. Utiliza ainda, as normas para definição de padrão de idioma e código do país. Respectivamente, a ISO 3166-1:2013, que “destina-se ao uso em qualquer aplicação que exija a expressão dos nomes de países atuais em formato codificado; inclui também diretrizes básicas para sua implementação e manutenção" (ISO, 2013) e ISO 639-3:2007 que contém códigos de idioma que compreendem identificadores de idioma (ISO, 2007).

Cada formulário pode ser aplicado apenas a coleções, não a itens digitais, individualmente. O formulário é atribuído a cada coleção no momento da submissão do primeiro item digital à mesma, no qual o serviço solicita mapeamento da coleção. A partir desse mapeamento, todos os itens digitais serão submetidos com os metadados contidos no formulário selecionado, não sendo possível a utilização de mais de um formulário dentro da mesma coleção (Shintaku e Meirelles, 2010). Os formulários desenvolvidos foram implementados conforme a Tabela 1 .

\footnotetext{
${ }^{1}$ https://wiki.duraspace.org/display/DSDOC6x/XMLUI+Configuration+and+Customization

${ }^{2} \mathrm{https} / / /$ wiki.duraspace.org/display/DSDOC6x/JSPUI+Configuration+and+Customization
} 
Tabela 1. Ocorrência de metadados nos formulários (fonte: elaboração do autor)

\begin{tabular}{|c|c|c|c|}
\hline $\begin{array}{l}\text { Segmento de Legislação } \\
\text { (Formualário B) }\end{array}$ & $\begin{array}{l}\text { Número de } \\
\text { ocorrência }\end{array}$ & $\begin{array}{l}\text { Segmento de Publicação } \\
\text { (Formulário A) }\end{array}$ & $\begin{array}{l}\text { Número de } \\
\text { ocorrência }\end{array}$ \\
\hline $\begin{array}{l}\text { dc.contributor.author - } \\
\text { Autor }\end{array}$ & 1 ou mais & $\begin{array}{l}\text { dc.contributor.author - } \\
\text { Autor }\end{array}$ & 1 ou mais \\
\hline dc.title - Título & 1 ou mais & dc.title - Título & 1 ou mais \\
\hline $\begin{array}{l}\text { dc.title.alternative - } \\
\text { Outros títulos }\end{array}$ & 1 ou mais & $\begin{array}{l}\text { dc.title.alternative - } \\
\text { Outros títulos }\end{array}$ & 1 ou mais \\
\hline $\begin{array}{l}\text { dc.date.created - Data de } \\
\text { assinatura }\end{array}$ & 1 ou mais & $\begin{array}{l}\text { dc.contributor.other - } \\
\text { Colaborador }\end{array}$ & 1 ou mais \\
\hline $\begin{array}{l}\text { dc.date.issued - Data de } \\
\text { publicação }\end{array}$ & 1 ou mais & dc.publisher - publicador & 1 ou mais \\
\hline $\begin{array}{l}\text { dc.description.abstract - } \\
\text { Ementa }\end{array}$ & 1 & $\begin{array}{l}\text { dc.date.issued - Data de } \\
\text { publicação }\end{array}$ & 1 \\
\hline dc.subject - Assunto & 1 & $\begin{array}{l}\text { dc.description.abstract - } \\
\text { Resumo }\end{array}$ & 1 \\
\hline dc.description - Notas & 1 ou mais & dc.subject - Assunto & 1 ou mais \\
\hline $\begin{array}{l}\text { dc.identifier.citation - } \\
\text { Fonte }\end{array}$ & 1 ou mais & dc.description - Notas & 1 ou mais \\
\hline dc.relation.uri - Link & 1 & $\begin{array}{l}\text { dc.identifier.citation - } \\
\text { Fonte }\end{array}$ & 1 \\
\hline $\begin{array}{l}\text { dc.relation - publicação } \\
\text { relacionada }\end{array}$ & 1 & dc.relation.uri - Link & 1 \\
\hline $\begin{array}{l}\text { dc.identifier.uri - } \\
\text { Identificador único }\end{array}$ & 1 ou mais & $\begin{array}{l}\text { dc.relation - publicação } \\
\text { relacionada }\end{array}$ & 1 ou mais \\
\hline dc.type - Tipo & 1 & $\begin{array}{l}\text { dc.identifier.uri - } \\
\text { Identificador único }\end{array}$ & 1 \\
\hline dc.rights - Licença & 1 ou mais & dc.type - Tipo & 1 ou mais \\
\hline dc.language - Idioma & 1 & dc.rights - Licença & 1 \\
\hline $\begin{array}{l}\text { dc.relation.inversionof - } \\
\text { Situação }\end{array}$ & 1 & dc.language - Idioma & 1 \\
\hline $\begin{array}{l}\text { dc.relation.isreplacedby - } \\
\text { Vide }\end{array}$ & 1 ou mais & & \\
\hline
\end{tabular}

\section{PROPOSTA DE OTIMIZAÇÃO DO FORMULÁRIO DE SUBMISSÃO}

Os sistemas de informação automatizados devem apresentar informações com qualidade, conforme Silva (2008). Para isso, as pessoas responsáveis, que são fontes de entradas de informações, não podem inserir informações equivocadas, pois estas podem gerar saídas inadequadas e dificultar o processo de busca e recuperação destas informações. Como características dos sistemas de informação, apresentam-se facilidade de uso, flexibilidade nos ajustamentos, confiabilidade nas informações geradas e rentabilidade de custo (Silva, 2008).

Para o acesso eficiente e preciso da informação para os usuários finais, que são aqueles que acessam as informações como produto, é importante que não haja dificuldades de operação da ferramenta, tanto para os próprios usuários, quanto para os profissionais que submetem os itens digitais ao sistema. A assertiva anterior demonstra a importância do trabalho realizado pelos profissionais que inserem dados, pois eles são responsáveis pela forma com que os produtos serão disponibilizados. Para que o usuário tenha acesso a informações de qualidade, é necessário que o processo pelo qual a informação será tratada e disponibilizada também seja feito com qualidade e seguindo um fluxo predefinido.

Após o desenvolvimento da aplicação, foram aprimorados os seguintes aspectos:

a) Todos os passos necessários para submissão foram agregados em única página;

b) A interface tornou-se mais clara, limpa e intuitiva, de acordo com os submissores da ferramenta; 
c) A ordem de exibição dos campos foi organizada de forma melhor atender as necessidades do ME;

d) Cada segmento do acervo do ME foi atendido com sua relação de metadados específica.

Testes funcionais foram realizados com os bibliotecários que operam o sistema para avaliar a efetividade das melhorias. Pôde-se confirmar que com a customização do formulário de submissão ocorreu otimização do tempo de realização da tarefa, favorecimento da visualização e identificação dos campos e reconhecimento de erros em um único ponto do sistema. Os testes foram realizados com 5 usuários, com níveis de experiência e formação diferentes com utilização de uma amostra de 100 itens digitais que foram inseridos de forma a avaliar as novas funcionalidades.

\section{PROPOSTA DE ARQUITETURA DA BIBLIOTECA DIGITAL}

A modelagem da arquitetura da aplicação teve como objetivo a interação entre os microsserviços de forma que as atribuições fossem divididas em operações atômicas em um modelo amplo, como é demonstrado na Figura 2.

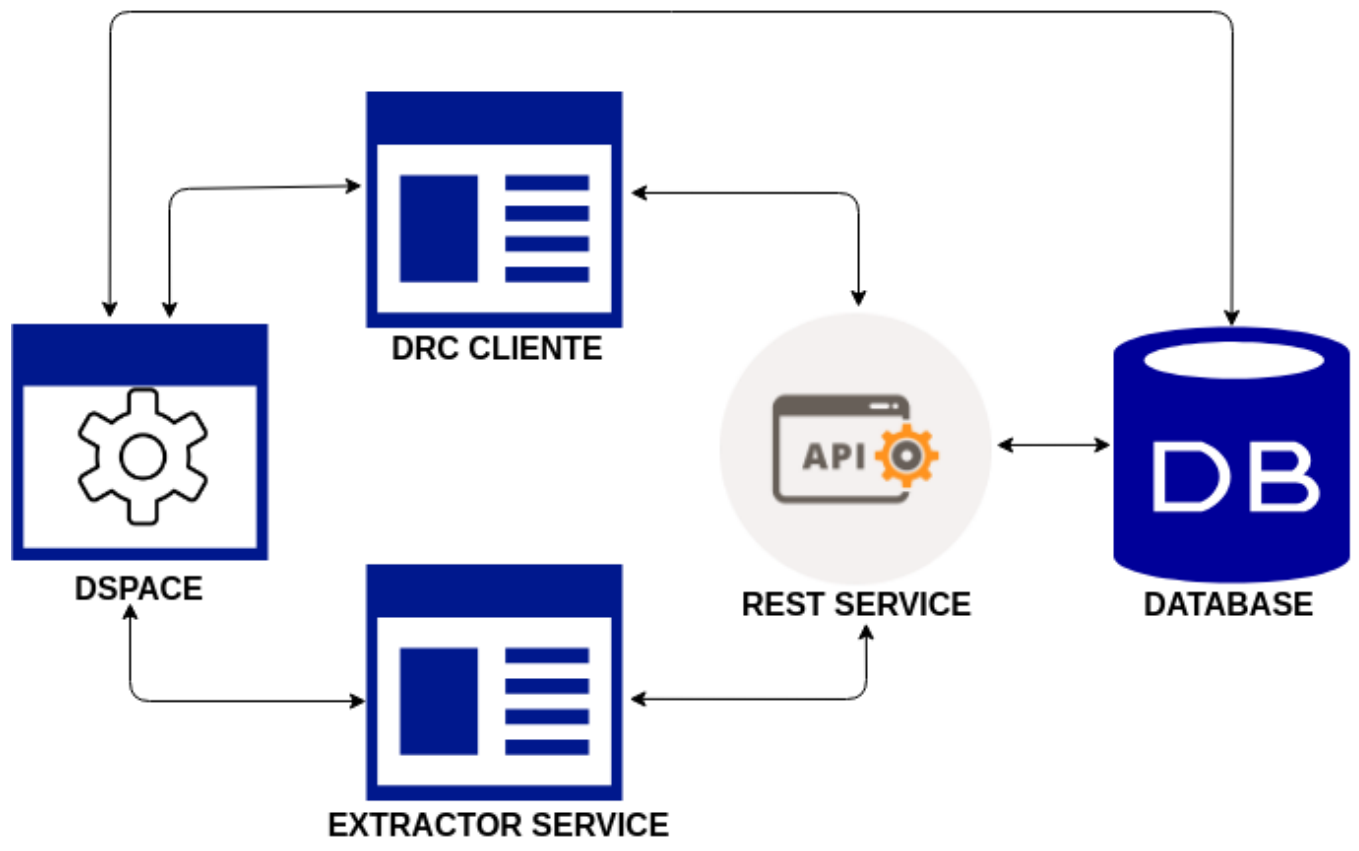

Figura 2. Modelagem da arquitetura para interoperabilidade da Biblioteca Digital do ME (fonte: elaboração do autor)

Os serviços fazem uso da REST API do DSpace para gerenciar atividades e efetuar inserções de novos itens digitais ao repositório da Biblioteca Digital. Estes serviços são requisitados a partir de links presentes na interface JSPUI.

Dois serviços foram modelados e desenvolvidos de forma que um efetua a inserção de novos itens digitais através da REST API do DSpace e o outro faz a extração de dados do Diário Oficial da União (DOU), para posterior inserção no DSpace.

O serviço de inserção, DRC service, é requisitado quando o usuário que possui credenciais para uma nova submissão requer esta atividade no sistema. A autenticação entre os agentes é feita a partir do envio de parâmetros necessários para que o DSpace envie um token de sessão que possibilita requisições seguras à REST API.

Desta forma, serviços interoperáveis podem se comunicar através de $\mathrm{JSON}^{3}$ para requisitar tarefas que se encontram em outros serviços. Este modo de requisição também pode ser chamado de requisição de recursos, pois uma tarefa é considerada um recurso no escopo geral de uma aplicação. O DSpace, na versão 6, possui uma implementação de serviço REST por meio do seu módulo de Interface de Programação de

\footnotetext{
${ }^{3}$ http://www.json.org/
} 
Aplicativos - REST API. Esta REST API disponibiliza várias rotas para execução de determinadas tarefas no Framework, como seleção de comunidades, coleções ou itens digitais, assim como criação, alteração e remoção desses e inserção de novos itens digitais.

O segmentos do acervo fazem com que a implementação dos serviços se distanciem no que tange ao formato da requisição de nova inserção ao repositório. O Extractor Service utiliza um código criptografado com os parâmetros de login, possibilitando maior segurança. O DRC Service não faz uso desta implementação.

Os requisitos principais para a modelagem e desenvolvimento da arquitetura da aplicação mantiveram-se com a seguinte prioridade:

a) Um serviço deverá efetuar inserção de itens digitais a partir da REST API do DSpace;

b) O formulário de inserção de novos metadados deverá ser customizado com itens obrigatórios, opcionais e metadados de mesmo tipo com mais de um valor;

c) O serviço deverá ser customizado em um único passo de formulário;

d) Um serviço de extração de metadados do Diário Oficial da União (DOU) deverá inserir novos itens digitais no DSpace a partir de sua REST API;

e) O tipo de item digital a ser inserido pelo serviço de extração deverá ser customizado para enviar para uma coleção específica nomeada "Diário Oficial da União".

Desta forma, foi possível dividir as funcionalidades para melhor adequar às necessidades da Biblioteca Digital do ME. A seguir, serão detalhadas as implementações dos serviços modelados.

\subsection{DSpace REST Client Service}

O serviço de submissão de itens digitais na Biblioteca do ME foi nomeado como DSpace REST Client - DRC Service, e constitui-se de um serviço escrito na linguagem de programação para Web PHP (Hypertext Preprocessor) na versão 7 (Welling e Thomson, 2003).

A implementação feita para o Serviço DRC efetua chamadas à REST API para consultar comunidades e suas respectivas coleções. Assim, é possível inserir um novo item digital através da REST API em uma rota específica e com parâmetros esperados, em conjunto com as credenciais do submissor do novo item. Deste modo, o Serviço DRC faz uso da REST API para inserir um novo item digital de forma que, o modelo de metadados inserido, esteja de acordo com a realidade da Biblioteca Digital do ME.

Uma Framework de desenvolvimento faz-se necessária para este tipo de arquitetura de aplicação no que tange a organização do projeto como um serviço que opera em contexto específico, executando uma tarefa pontual a que foi proposto, e global, fazendo parte do contexto da aplicação em termos gerais. Neste sentido, a Framework Yii (Safronov e Winesett, 2014), em sua segunda versão, foi utilizada para o desenvolvimento do DRC Service, o que fez com que o processo de desenvolvimento do serviço fosse acelerado e inseridos padrões de projeto de software em sua implementação.

\subsection{Modelagem do Serviço}

As alterações implementadas no formulário de submissão possibilitam que os campos recebam nova referência, ordenação e indicação de repetitividade e obrigatoriedade. A característica de repetitividade foi inserida para proporcionar a inserção dos valores na quantidade necessária para um mesmo metadado mantendo a lógica criada já existente no DSpace. Os nomes designados aos campos na customização do formulário, são exibidos também no registro simples do item digital para o usuário para que a informação seja percebida com maior facilidade.

A renderização do formulário customizado ocorre a partir da leitura de um arquivo presente no sistema, chamado "input-form.xml.", no qual são mapeadas todas as coleções de acordo com o seu respectivo formulário. Após o preenchimento dos dados do formulário, os mesmos são enviados para o serviço RestDspace, responsável pela abstração das regras de negócio do sistema.

A partir desse mapeamento, todos os itens digitais serão submetidos juntamente com os respectivos metadados, determinados conforme o formulário selecionado. Uma das principais motivações para a construção desta arquitetura de aplicação é que seriam indispensáveis alterações no código do Framework para efetuar as alterações necessárias para a Biblioteca Digital do ME no próprio DSpace. Optou-se por não realizar alterações no código interno do sistema para possibilitar que atualizações sejam efetuadas em lançamentos de novas versões, dispensando a remodelagem das configurações já realizadas a partir da versão 6 . 


\subsection{Modelo de Processo de Inserção}

O processo de inserção de novos itens digitais do DSpace padrão é dividido em cinco tarefas. No entanto, foi constatado pelos bibliotecários do ME que esta divisão desfavorece a agilidade na inserção de grandes quantidades de itens digitais. Neste sentido, o serviço de inserção proposto reduz as tarefas de cinco para uma, racionaliza o processo de inserção, diminui o tempo gasto em uma nova submissão e melhora a produtividade dos submissores. O processo proposto pode ser visualizado na Figura 3.

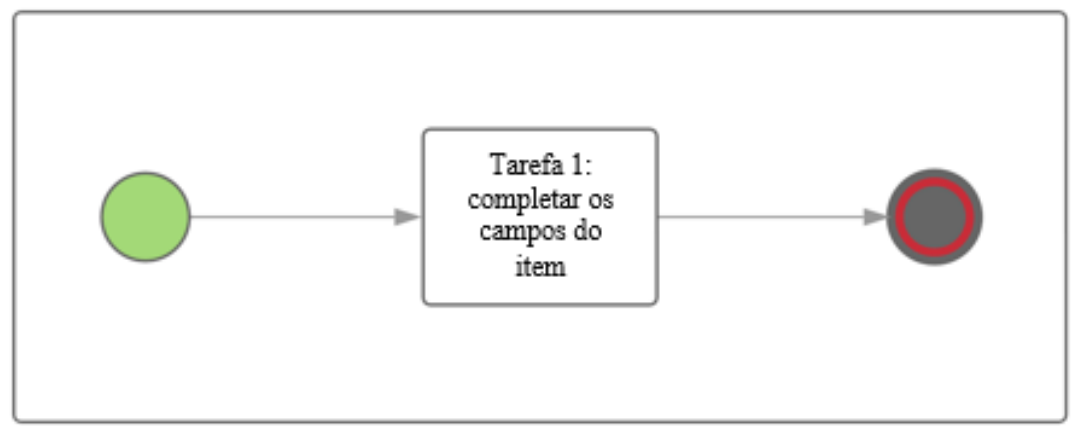

Figura 3. Modelo de processo de inserção proposto (fonte: elaboração do autor)

\section{RESULTADOS E DISCUSSÕES}

A Biblioteca Digital do ME foi desenvolvida a partir da arquitetura de microsserviços, na qual o DSpace é visto como um serviço dividido entre módulos, tais como REST API e JSPUI, e os microsserviços de auxílio customizados e desenvolvidos neste trabalho. Como modo alternativo de arquitetura para sistemas, possibilitou a estruturação da modelagem da arquitetura, de forma que dois serviços foram desenvolvidos. O primeiro serviço com objetivo de inserção de novos itens digitais, a partir de um formulário em um microsserviço que segmenta a estrutura de metadados em dois conjuntos distintos. O segundo serviço com o objetivo de extrair metadados de documentos publicados pela Imprensa Nacional no Diário Oficial da União e inserir na Biblioteca Digital do ME a partir da REST API do DSpace.

A arquitetura modelada encontra-se em fase de avaliação, com 13 comunidades, divididas entre áreas de interesse para a instituição, 7 subcomunidades, 73 coleções, relacionadas a essas comunidades e/ou subcomunidades, e 729 itens digitais, totalizando 40 gigabytes de dados.

\section{CONCLUSÕES E TRABALHOS FUTUROS}

O principal objetivo deste trabalho foi possibilitar a customização da plataforma DSpace para atender às necessidades do ME e efetuar sua divulgação. Nesse sentido, a plataforma modelada encontra-se em execução em ambiente de produção para gerenciamento dos bibliotecários da instituição. A primeira versão da aplicação encontra-se em fase de produção, também, pelos bibliotecários, e o desenvolvimento da segunda versão está em fase de modelagem, a partir dos novos requisitos demandados, com base no direcionamento desta primeira versão.

Com a customização da interface JSPUI, foi possível a adequação da Biblioteca Digital do ME ao padrão utilizado pelo Governo Brasileiro. No entanto, a Biblioteca Digital do ME ainda carece de uma análise de usabilidade baseada em heurísticas de usabilidade para aplicações Web e, desta forma, é proposta de trabalhos futuros, assim como a inserção de modelo de árvores de decisão, o qual já se encontra em andamento, para extração de entidades nomeadas no microsserviço de extração de itens inseridos em repositórios de dados não estruturados como por exemplo o Diário Oficial da União (DOU). 


\section{AGRADECIMENTO}

Os autores agradecem o apoio das Agências brasileiras de pesquisa, desenvolvimento e inovação CNPq (Projeto INCT SegCiber 465741/2014-2), CAPES (Projetos FORTE 23038.007604/2014-69 e PROBRAL 88887.144009/2017-00) e FAPDF (Projetos UIoT 0193.001366/2016 e SSDDC 0193. 001365/2016), bem como o suporte do Laboratório LATITUDE/UnB (Projeto SDN 23106. 099441/2016-43), e as cooperações com o Ministério da Economia (TEDs DIPLA 005/2016 e ENAP 083/2016) e o Gabinete de Segurança Institucional da Presidência da República (TED 002/2017).

\section{REFERÊNCIAS}

Behr, A. R. and Ferreira, M. K., 2016. Customizações no DSpace para melhorar interação do usuário no repositório digital Lume. Anais de Workshop de Tecnologia de Informação e Comunicação das Instituições Federais de Ensino Superior. Gramado, Brasil.

Costa, J. S. B. D. et al., 2015. Implementações no DSpace para disponibilização de recursos educacionais no repositório digital da universidade federal do rio grande do sul. Anais de Conferencia de Directores de Tecnología de Información. Viña del Mar, Chile, não paginado.

International Organization for Standardization. ISO 15836-1:2017. 2017.

International Organization for Standardization. ISO 3166-1:2013. 2013.

International Organization for Standardization. ISO 639-3:2007. 2007.

International Organization for Standardization. ISO 8601-1:2019. 2019.

Safronov, M. and Winesett, J., 2014. Web Application Development with Yii 2 and PHP. Packt Publishing Ltd, Birminggham, United Kingdom.

Shintaku, M. and Meirelles, R.F., 2010. Manual do DSpace: administração de repositórios. EDUFBA, Salvador, Brasil.

Shintaku, M. and Vechiato, F.L., 2018. Histórico do uso do DSpace no brasil com foco na tecnologia. Revista Informação na Sociedade Contemporânea, Vol. 2, No. 1, pp. 1-16.

Silva, P. M., 2008. Sistemas de informação em bibliotecas: o comportamento dos usuários e bibliotecários frente às novas tecnologias de informação. RDBCI: Revista Digital de Biblioteconomia e Ciência da Informação, Vol. 6, No. 1, pp 1-24.

Viana, C. L. D. M., Márdero Arellano, M. Á., and Shintaku, M., 2005. Repositórios institucionais em ciência e tecnologia: uma experiência de customização do DSpace. Anais de Simpósio Internacional de Bibliotecas Digitais. São Paulo, Brasil, não paginado.

Wei, Z., 2011. Research on the Application of Open Source Software in Digital Library. Procedia Engineering, Vol. 15, pp. 1662-1667.

Weibel, S. L. and Koch, T., 2000. The Dublin Core Metadata Initiative. D-Lib Magazine, Vol. 6, No. 12, pp. 1082-9873.

Welling, L. and Thomson, L., 2003. PHP and MySQL Web development. Sams Publishing. Carmel, United States. 